\title{
The Effects of Two Different Physical Activity Organizing Methods on the Executive Functions of the Children with Developmental Coordination Disorder
}

\author{
Hadi Moradi ${ }^{*}$, Abolfazl Shayan Nooshabadi² \\ ${ }^{1}$ Department of Motor Behavior, Faculty of Sport Sciences, Ferdowsi University of Mashhad, Mashhad, Iran \\ ${ }^{2}$ Department of Sport Sciences, Facaulty of Literature and Humanities, Jahrom University, Jahrom, Iran
}

\begin{tabular}{lll}
\hline & Article Info: & \\
\hline Received: 19 Feb 2018 & Revised: 7 Jun 2018 & Accepted: 15 Jul 2018
\end{tabular}

\section{A BSTRACT}

Introduction: Since the people with developmental coordination disorder are weak in executive functions, the present study was aimed to investigate the effect of two different physical activity organizing methods on the executive functions of children with developmental coordination disorder. Materials and Methods: Among the children with developmental coordination disorder that were eligible to enter the study, 45 subjects were randomly selected and after completing Conner's Executive Functioning Scale as pretest, randomly divided into three groups; random practice group (15 subjects), blocked practice group (15 subjects), and control group (15 subjects). Then, the two interventional groups performed physical practices with different underlying interactions for 8 weeks, with three 45 -minute sessions each week. At the end, all the subjects underwent a posttest by executive functioning scale. Results: After the interventions, the results showed a significant improvement in the executive functions of the two intervention groups. However, no significant improvement was observed in the executive functions of the control group. In addition, a significant difference between random and blocked practice groups was observed. However, the random practice group had a greater improvement in the sensory-motor functions. Conclusion: According to our data, random physical practices can be considered as an ideal method to improve executive functions of the children with developmental coordination disorder.

\section{Key words:}

1. Executive Function

2. Exercise

3. Child

*Corresponding Author: Hadi Moradi

E-mail: hadi.moradi@stu.um.ac.ir 


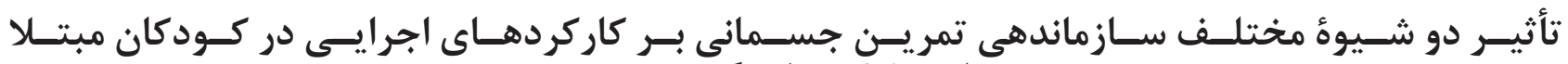

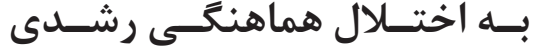

\author{
هادى مرادى ا:" ابوالفضل شايان نوش آبادى \\ اكروه رفتار حركتى، دانشكده علوم ورزشى، دانشكاه فردوسى مشهد، مشهد، ايران

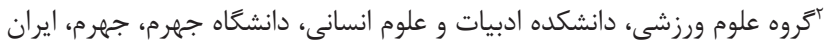

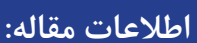

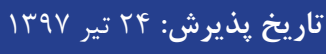

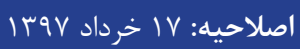

تاريخ دريافت: · ب بهمن عqسا

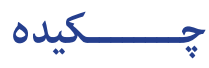

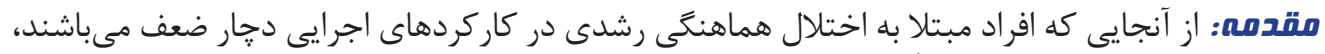

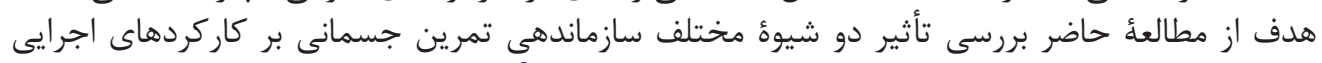

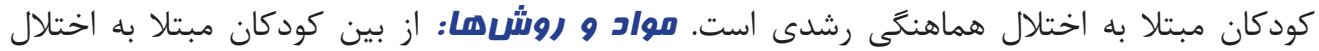

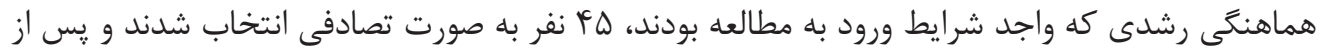

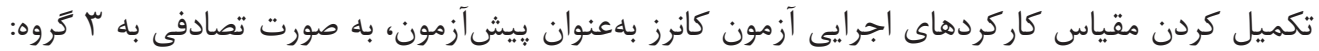

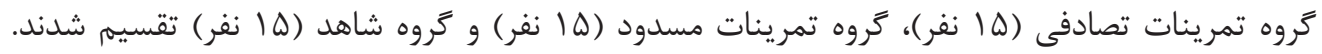

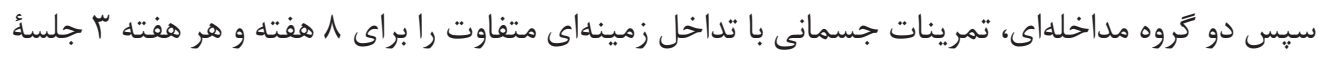

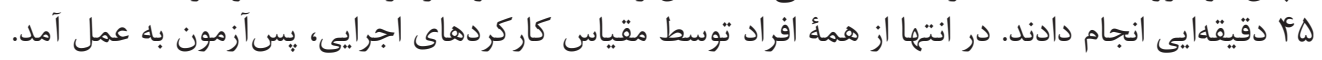

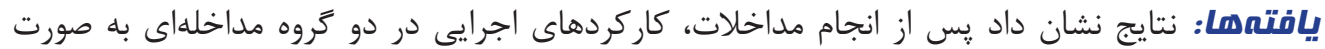

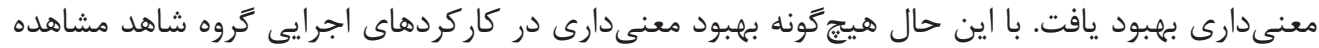

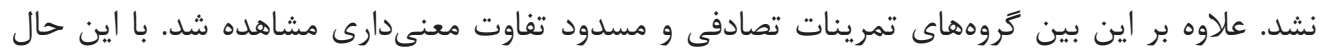

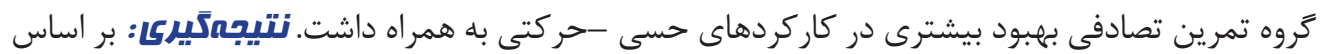

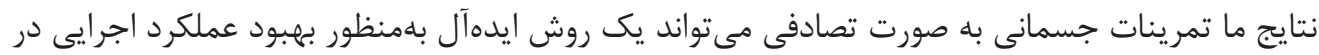

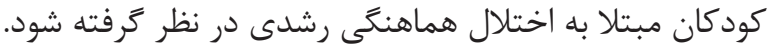

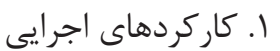

r.

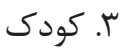

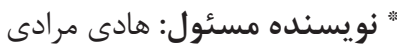

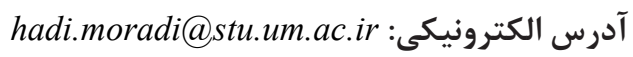




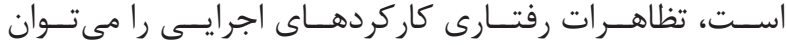

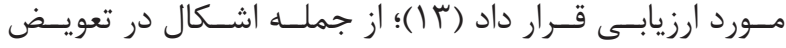

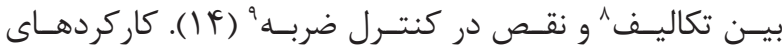

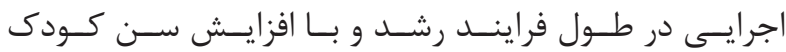

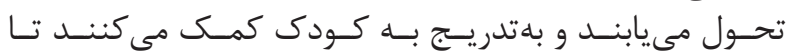

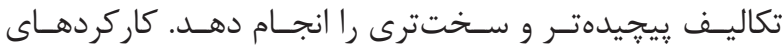

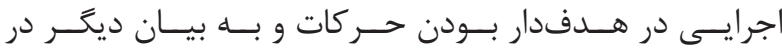

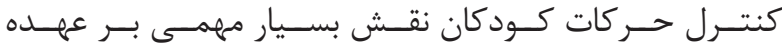

دارنــد (ه) (1).

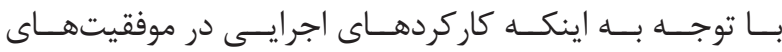

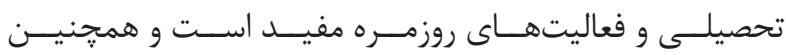

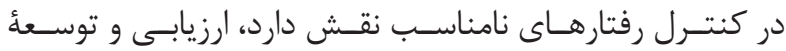

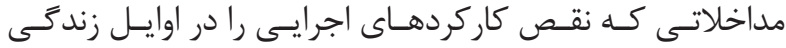

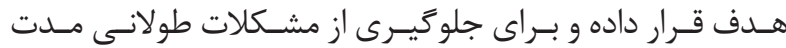

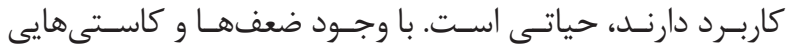

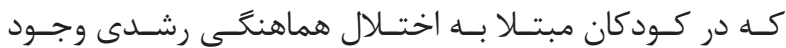

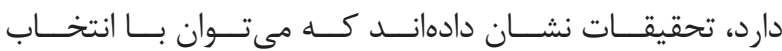

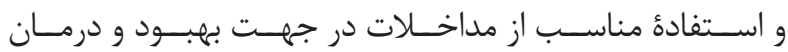

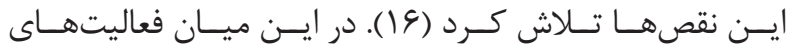

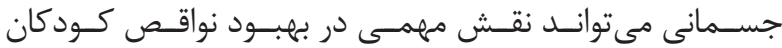

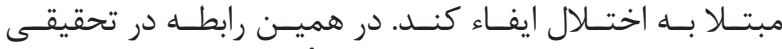

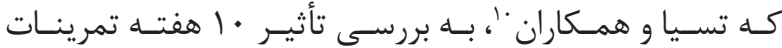

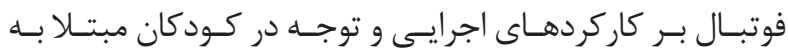

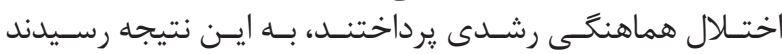

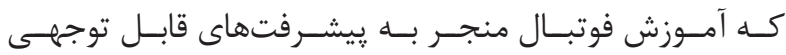

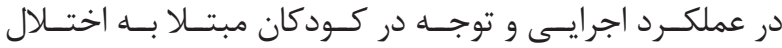

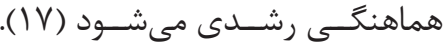

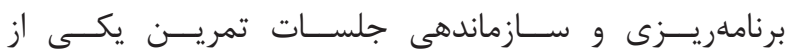

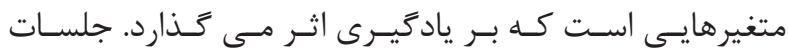

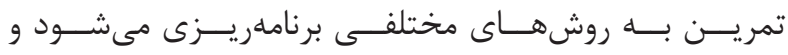

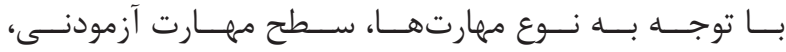

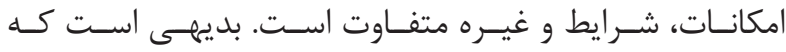

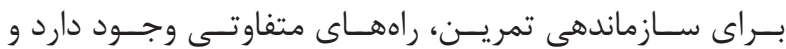

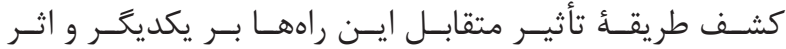

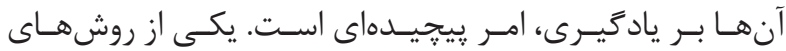

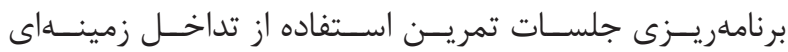

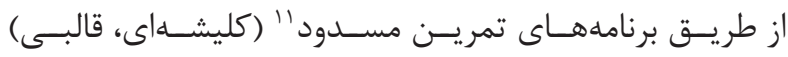

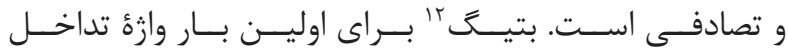

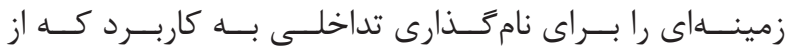

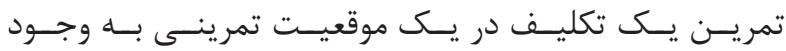

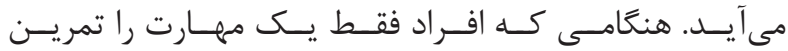

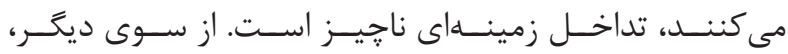

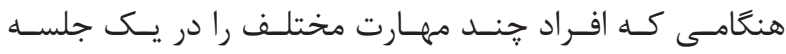

\footnotetext{
${ }^{1}$ Developmental coordination disorder

${ }^{2}$ Minimal brain dysfunction

${ }^{3}$ Perceptual motor dysfunction

${ }^{4}$ Developmental apraxia

${ }^{5}$ Physical awkwardness

${ }^{6}$ Diagnostic and statistical manual of mental disorders-fifth edition
}

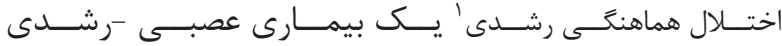

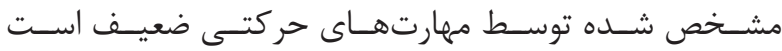

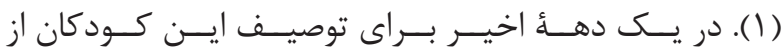

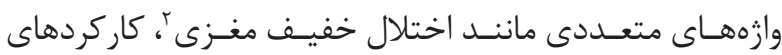

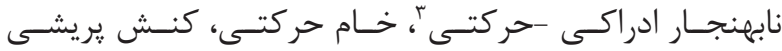

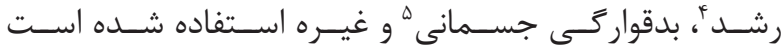

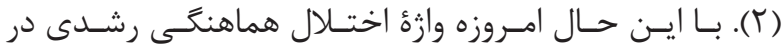

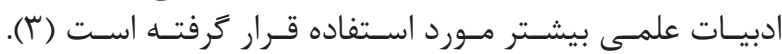

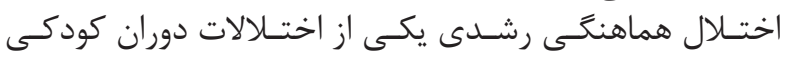

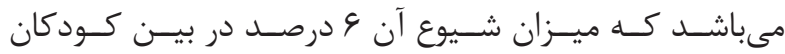

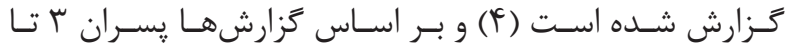

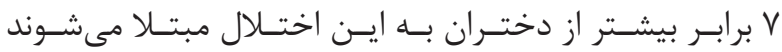

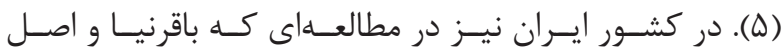

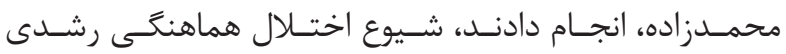

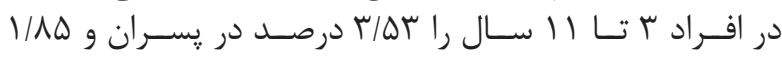

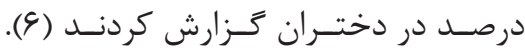

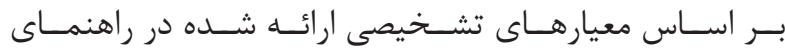

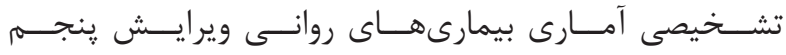

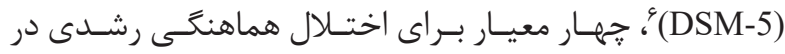

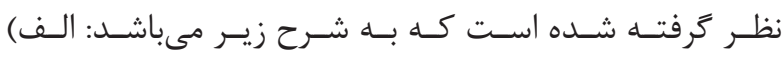

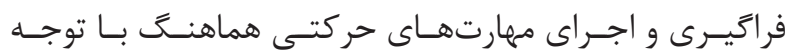

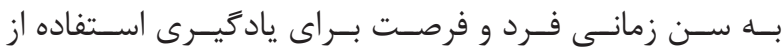

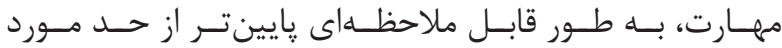

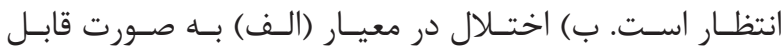

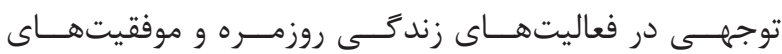

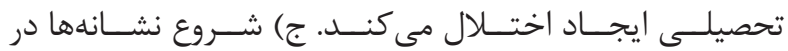

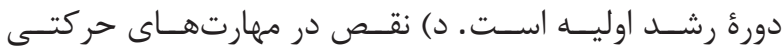

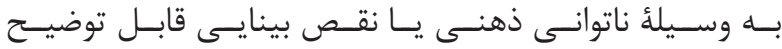

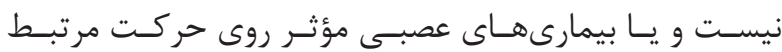

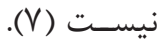

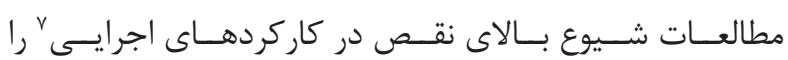

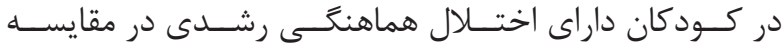

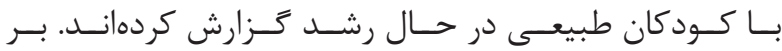

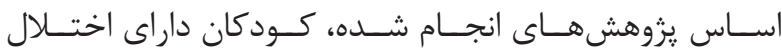

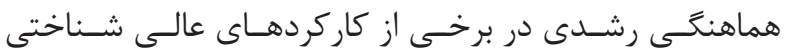

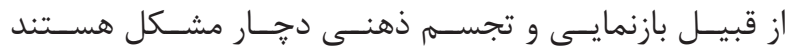

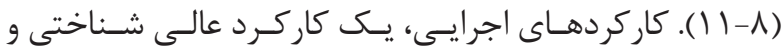

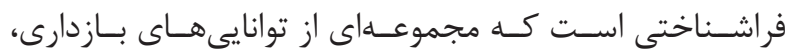

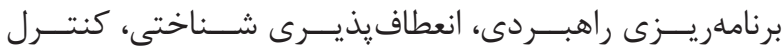

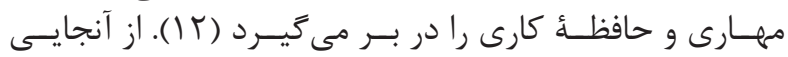

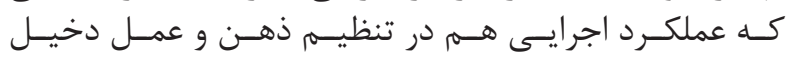

\footnotetext{
${ }^{7}$ Executive functions

${ }^{8}$ Difficulty switching between tasks

${ }^{9}$ Lack of impulse control

${ }^{10}$ Tsai

${ }^{11}$ Contextual interference

${ }^{12}$ Battig
} 


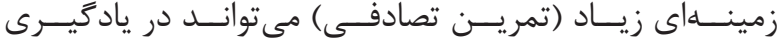

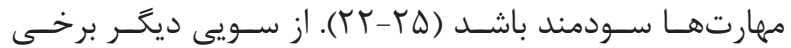

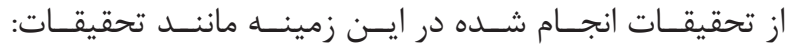

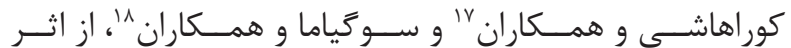

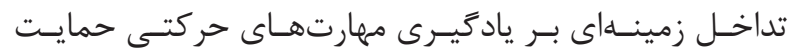

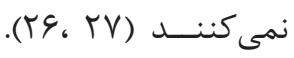

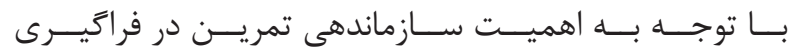

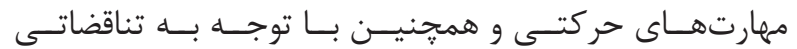

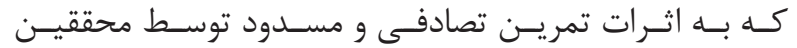

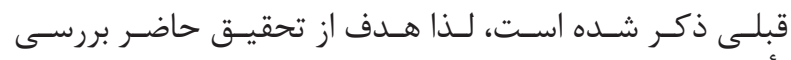

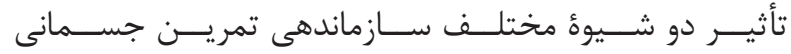

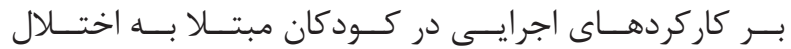

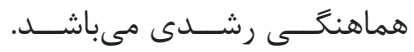

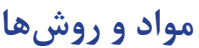

روش يثروهش، جامعلُ آمارى و نمونه

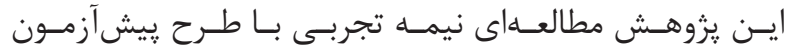

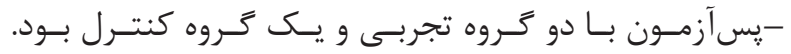

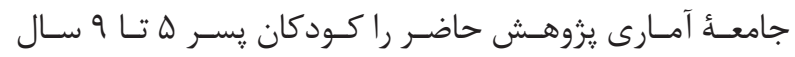

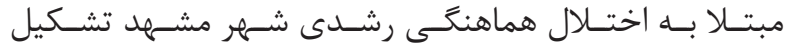

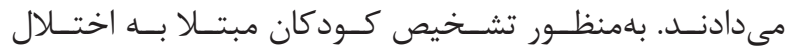

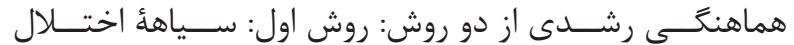

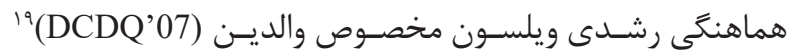

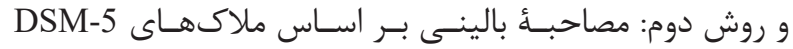

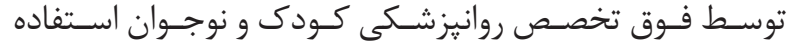

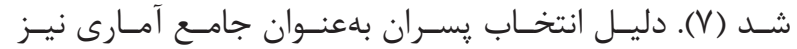

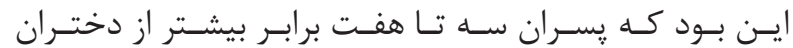

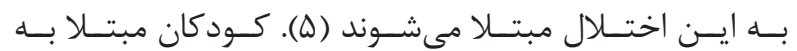

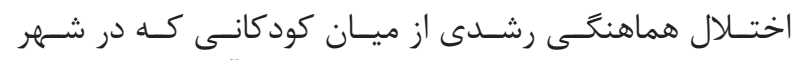

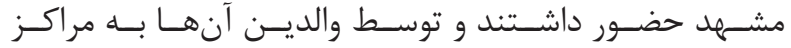

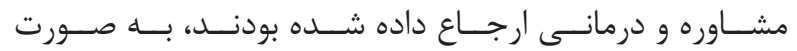

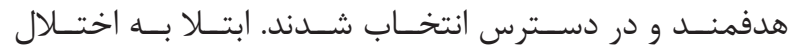

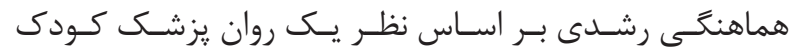

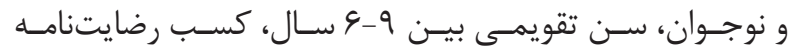

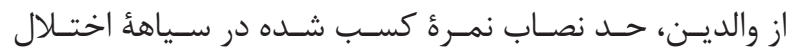

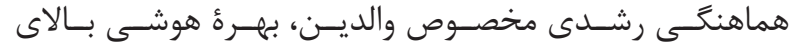

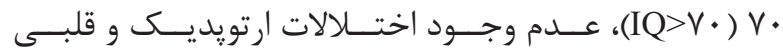

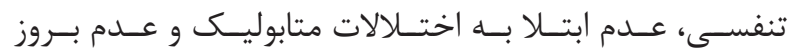

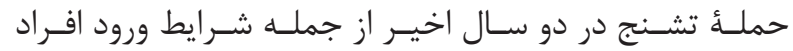

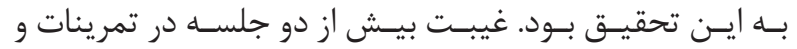

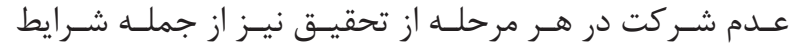

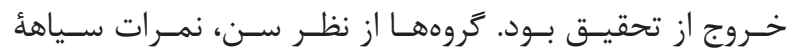

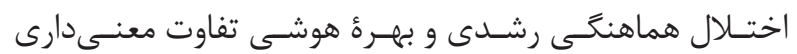
بـا همديخــر نداشـتـند.

\footnotetext{
${ }^{13}$ Random practice

${ }^{14}$ Porter

${ }^{15}$ Jones

${ }^{16}$ Russell and Newell
}

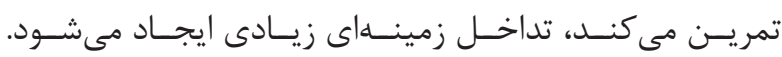

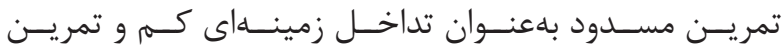

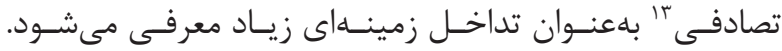

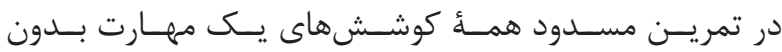

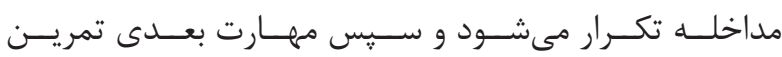

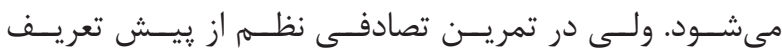

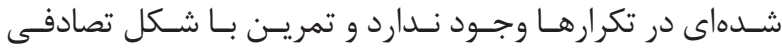

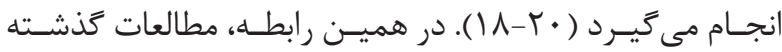

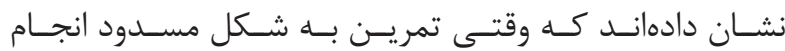

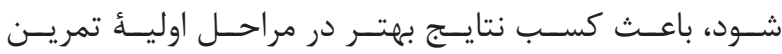

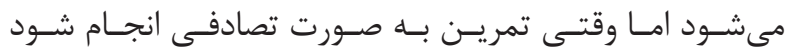

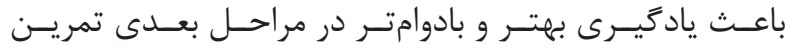

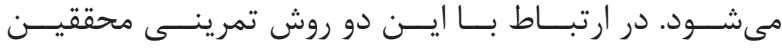

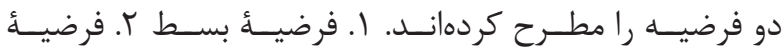

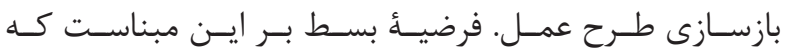

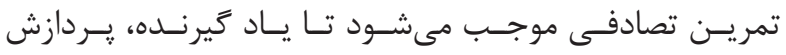

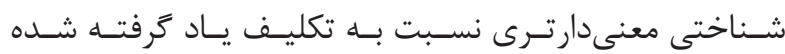

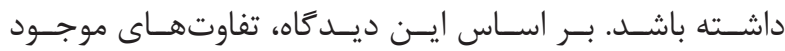

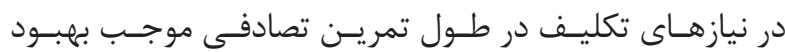

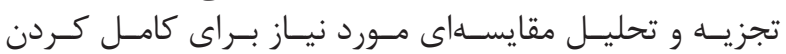

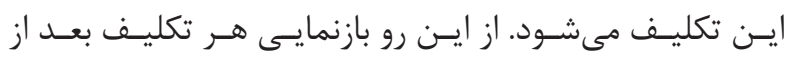

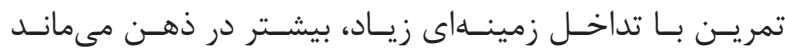

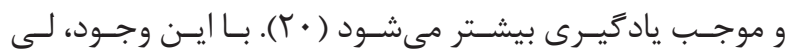

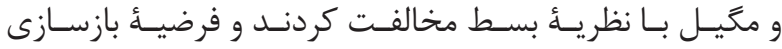

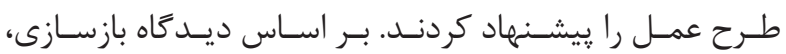

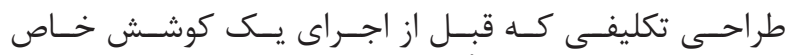

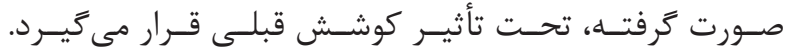

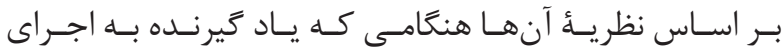

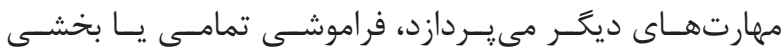

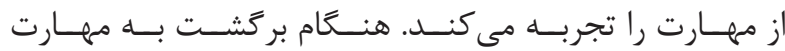

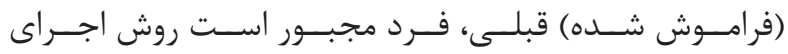

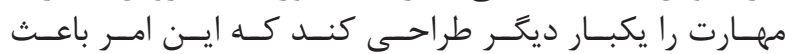

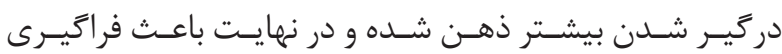

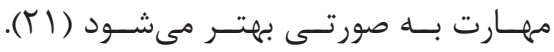

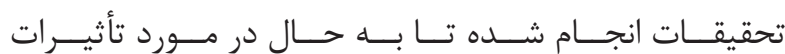

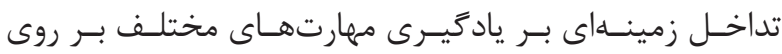

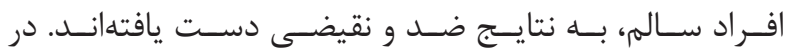

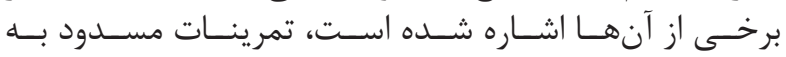

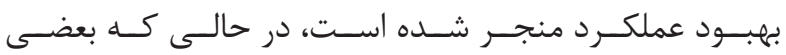

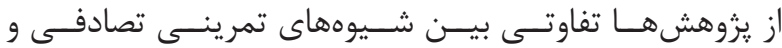

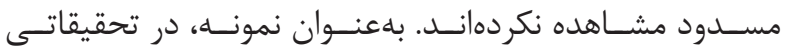

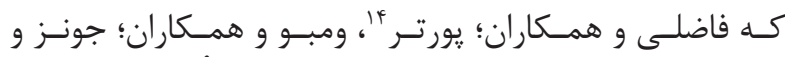

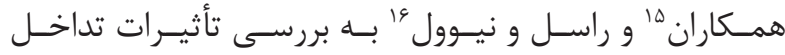

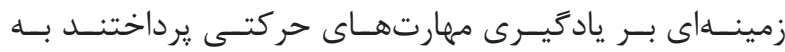

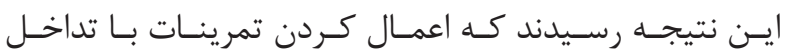

\footnotetext{
${ }^{17}$ Kurahashi

${ }^{18}$ Sugiyama

${ }^{19}$ Developmental coordination disorder questionnare
} 


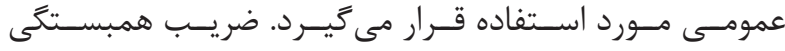

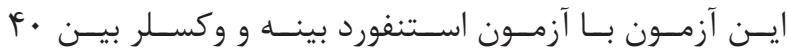

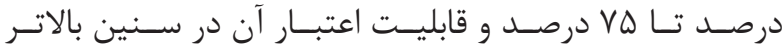

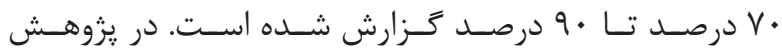

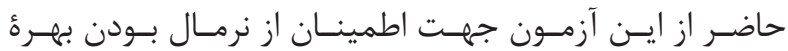

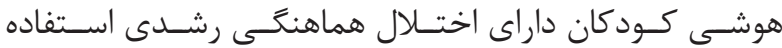

شــد (r人).

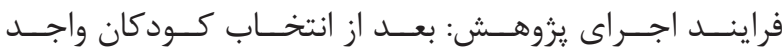

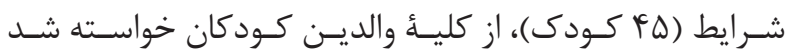

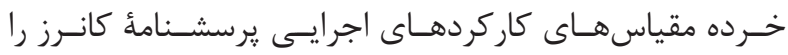

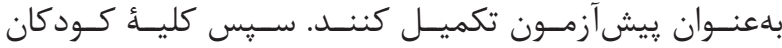

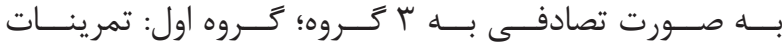

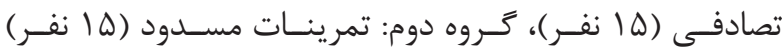

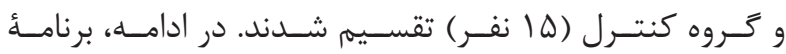

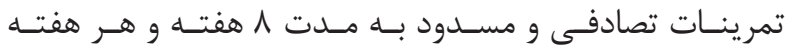

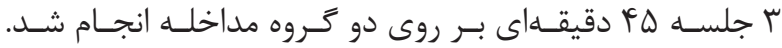

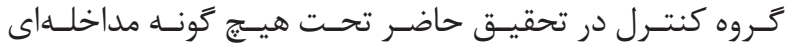

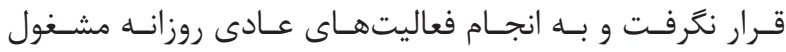

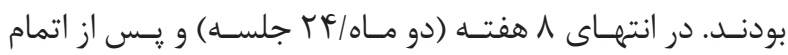

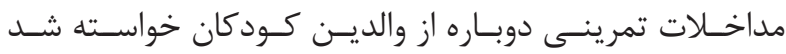

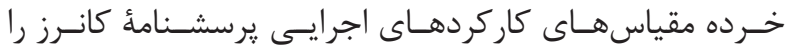

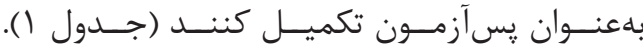

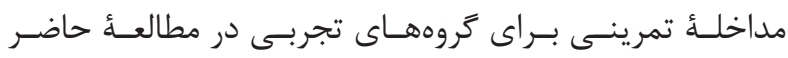

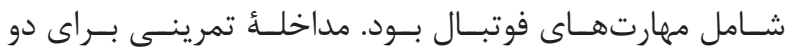

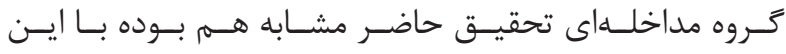

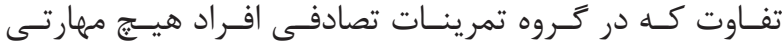

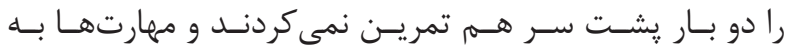

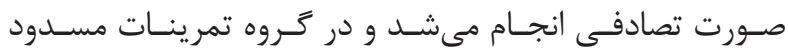

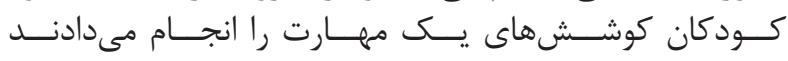

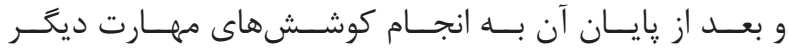

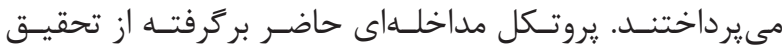

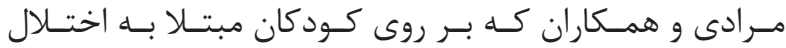

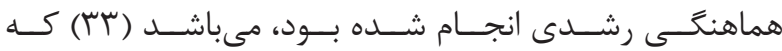

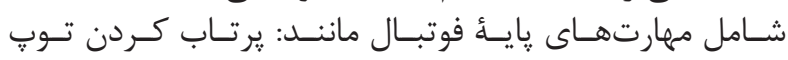

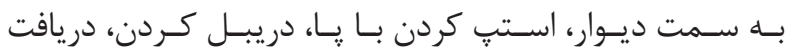

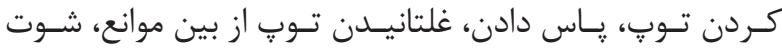

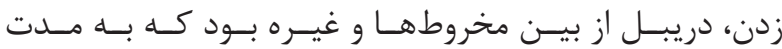

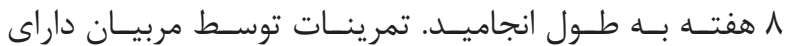

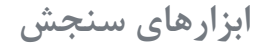

ا- آزمــون عصــب -روانشـــناختى كانـــرز: ايـن آزمــون

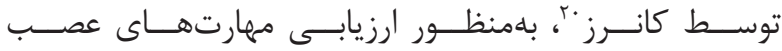

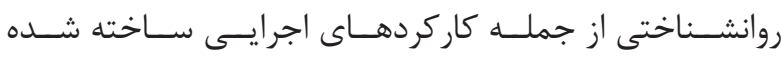

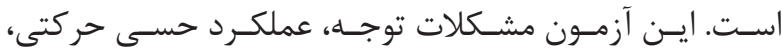

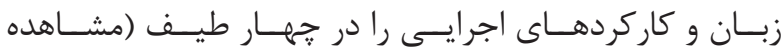

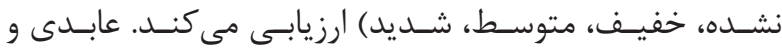

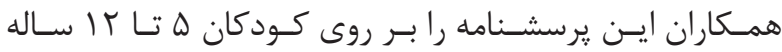

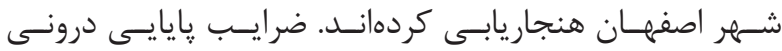

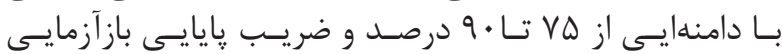

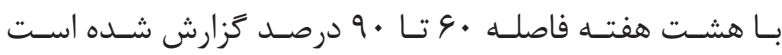

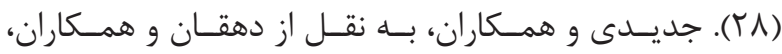

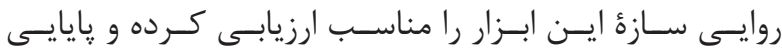

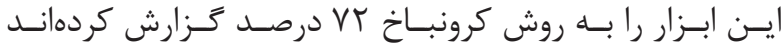

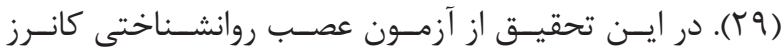

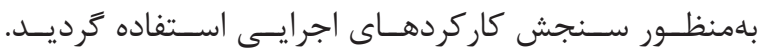

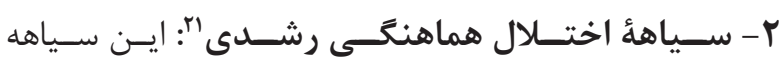

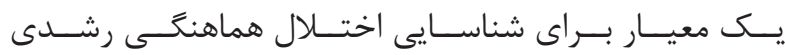

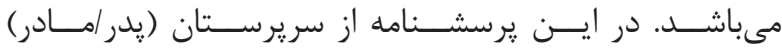

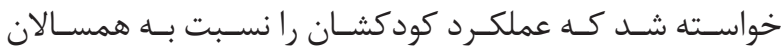

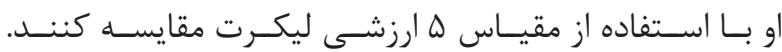

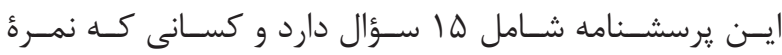

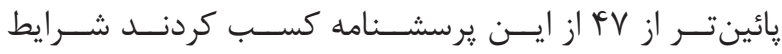

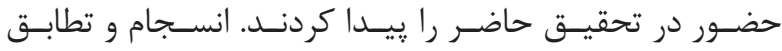

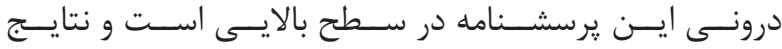

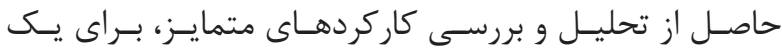

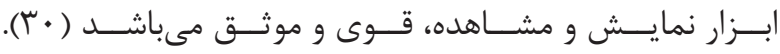

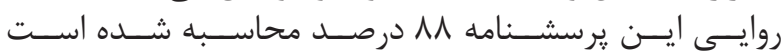

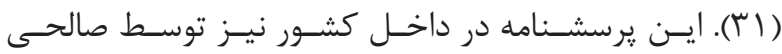

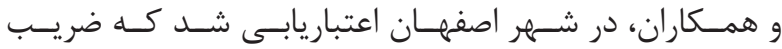

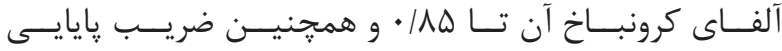

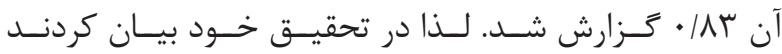

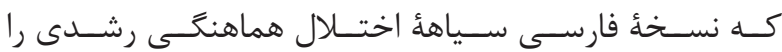

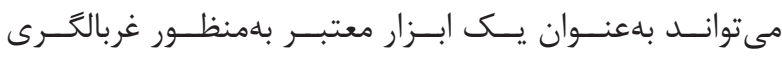

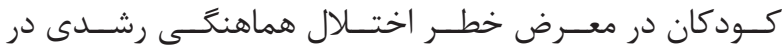

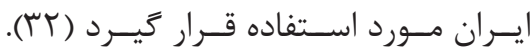

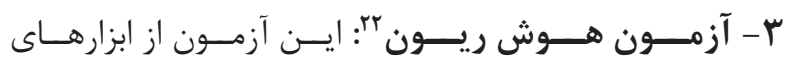

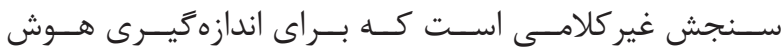

\begin{tabular}{|c|c|c|c|}
\hline بس از مداخله (•9 روز) & مداخله & 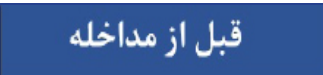 & 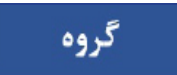 \\
\hline ارزيابى كاركردهاى اجرايى & تمرينات جسمانى به شكل مسدود & ارزيابى كاركر دهاى اجرايى & تمرين مسدود \\
\hline ارزيابى كاركردهاى اجرايى & تمرينات جسمانى به شكل تصادفى & ارزيابى كاركر دهاى اجرايى & تمرين تصادفى \\
\hline ارزيابى كاركردهاى اجرايى & ------------------- & ارزيابى كاركر دهاى اجرايى & 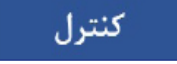 \\
\hline
\end{tabular}

\footnotetext{
${ }^{20}$ Canners

${ }^{21}$ Wilson

${ }^{22}$ Raven
} 


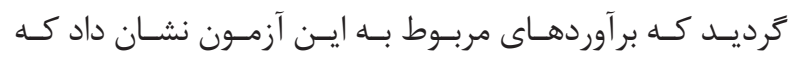

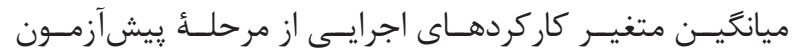

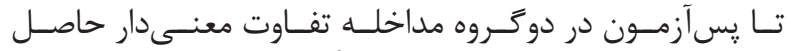

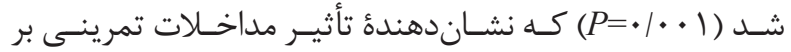

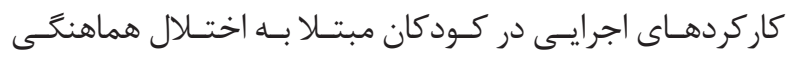

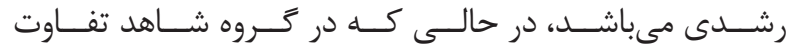

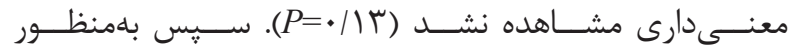

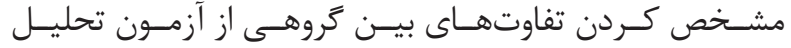

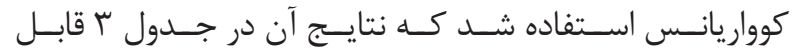

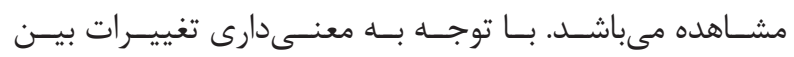

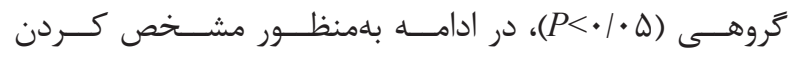

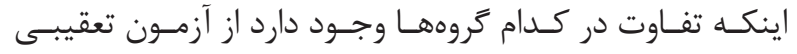

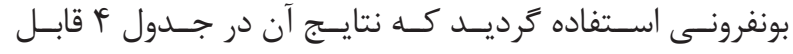
مشــاهده اسـتـ

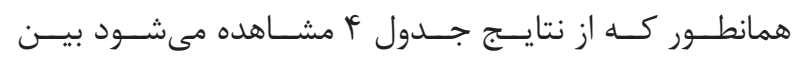

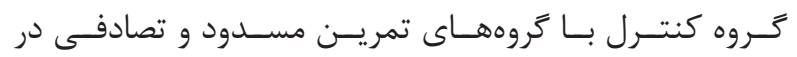

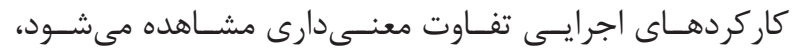

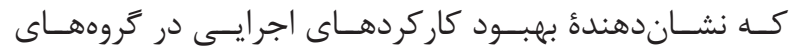

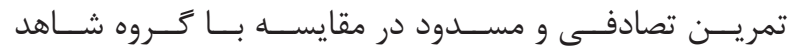

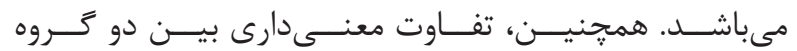

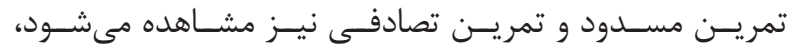

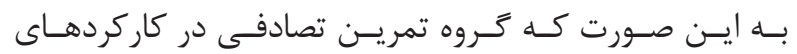

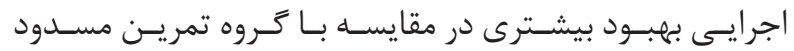
بــه دسـت آوردنـــ (نمـودار (1).

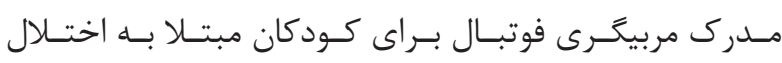

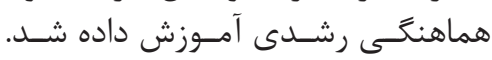

تجزيه و تحليل دادهها

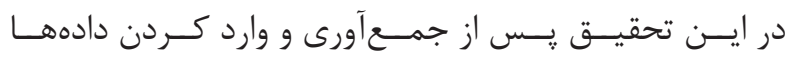

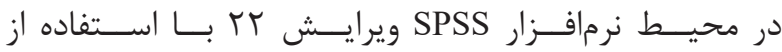

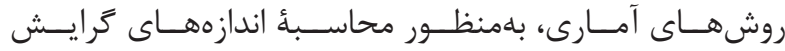

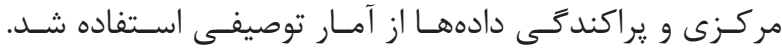

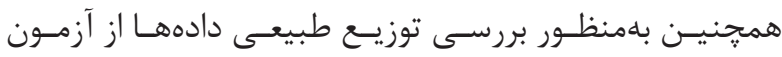

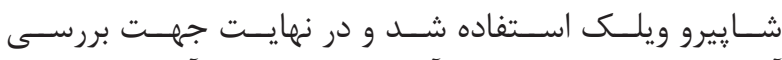

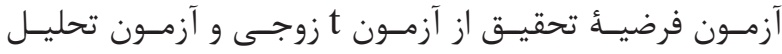

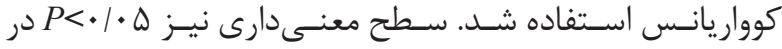

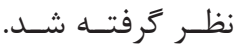

يافتهها

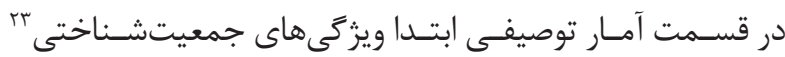

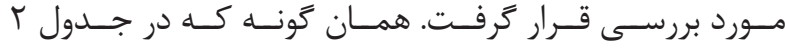

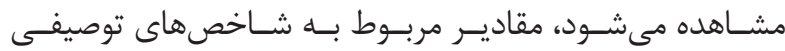

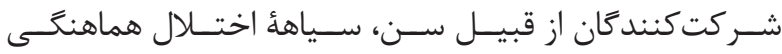

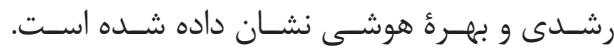

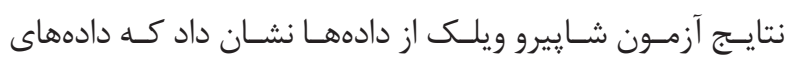

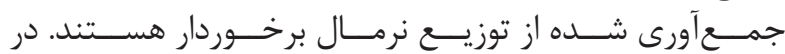

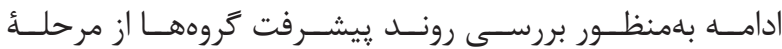

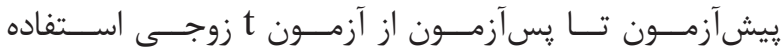

جدول r - بِيش آزمون و پِسآزمون متغيرهاى تحقيق و مشخصات شركت كنندكًان در كروههاى كنترل و مداخله.

\begin{tabular}{|c|c|c|c|}
\hline 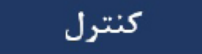 & تمرين تصادفى & تمرين مسدود & 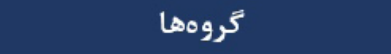 \\
\hline ل ال انفر & ل ال الفر & ل ال الفر & تعداد \\
\hline $4 / \cdot 9 \pm V / \Delta$ & $\Delta / \Lambda V \pm I \Delta / \Lambda$ & $\Delta / \& r \pm 9 / \&$ & سن (سال) \\
\hline$r \Delta / 9 r \pm V / .9$ & $r q / f+ \pm v / \cdot r$ & $r v / r+ \pm v / \Delta r$ & مخياهُ اختلال هماهنگى رشلى \\
\hline$\Lambda G / F V \pm F / K \Delta$ & $\Lambda q / \cdot V \pm r / v \wedge$ & $\Lambda \Delta / \Lambda \cdot \pm F / r q$ & بهرء هوشى رنتى ريون (IQ) \\
\hline
\end{tabular}

جدول بـ- برآورد تحليل كووار يانس بهمنظور مقايسٔ ميانكَين متغير كاركردهاى اجرايى در بين كروههاى تحقيق.

\begin{tabular}{|c|c|c|c|c|c|c|c|}
\hline اندازةٌ اثر & معنى دارى & آمارة F F F & ميانكين مربعات & DF & مجموع مربعات & آماره & متغيير \\
\hline.$/ 89$ & $\cdot 1+.+1$ & $19 r / r \cdot V$ & rrA/DVF & 1 & rR/ $/ \Delta V F$ & ييش آزمون & \\
\hline$\cdot / \Delta V$ & $\cdot 1+.+1$ & rV/ $\Delta G G$ & $\Delta \Delta / F \Phi T$ & r & 11.1948 & كروه & كاركر دهاى اجرايى \\
\hline - & - & - & $r /+1$ & fi & AT/FqR & خطا & \\
\hline
\end{tabular}

\begin{tabular}{|c|c|c|c|c|}
\hline معنى دارى & انحر اف استانفارد & اختلاف ميانگين & تروه & تروه \\
\hline$\cdot 1++r$ & . & $-1 / 9 F \Delta$ & تمرين مســود & \multirow{2}{*}{ تروه كنترل } \\
\hline$+1++1$ & - $|\Delta F|$ & $-f / \cdot I V$ & تمرين تصادفى & \\
\hline$\bullet 1 \cdot+1$ &.$/ \Delta T$. & $-r / \cdot V T$ & تمرين تصادفى & تمرين مسدود \\
\hline
\end{tabular}

${ }^{23}$ Demographic 


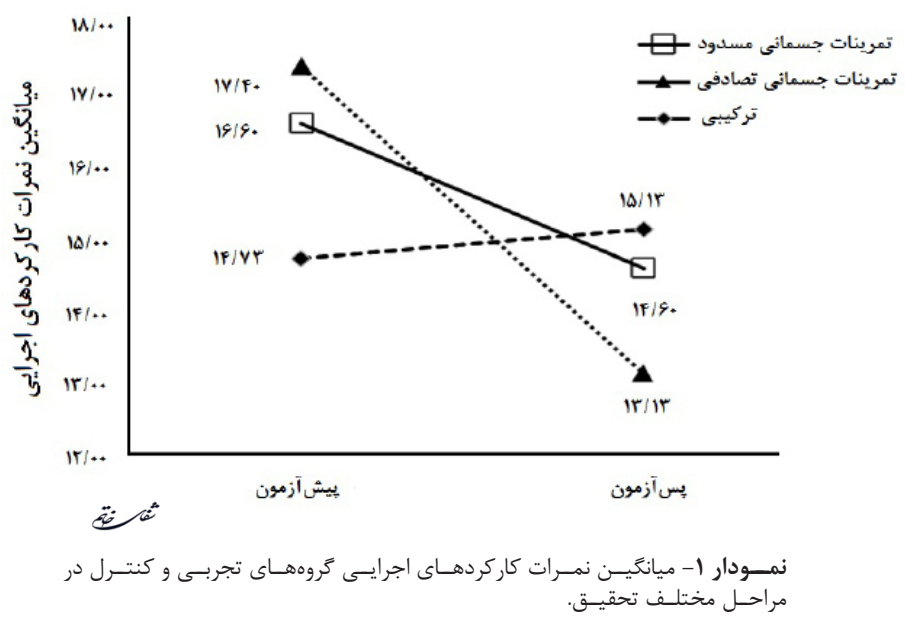

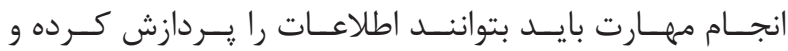
بحث و نتيجه كَيرى

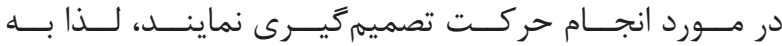

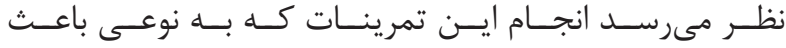

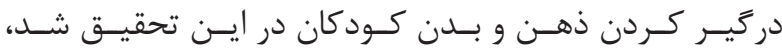

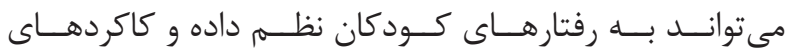

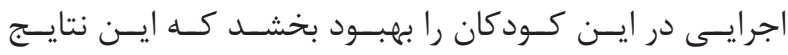

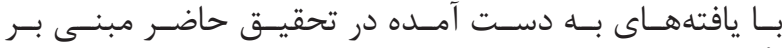

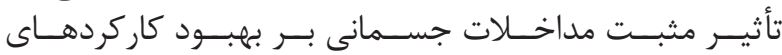

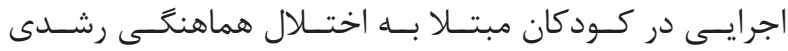

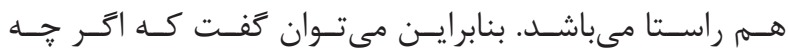

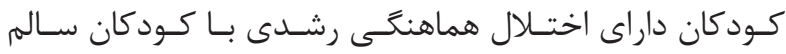

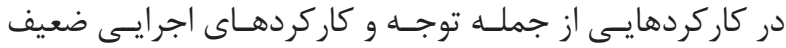

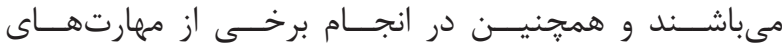

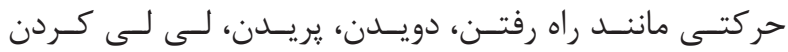

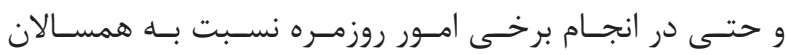

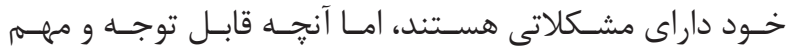

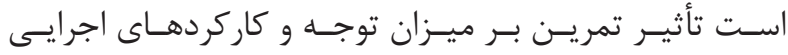

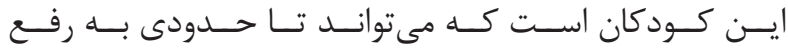

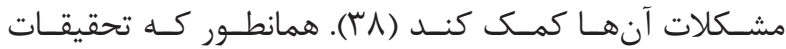

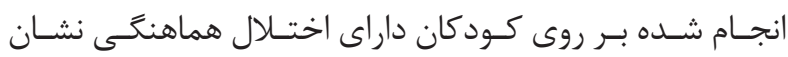

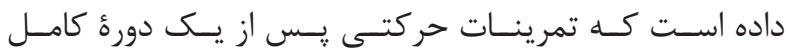

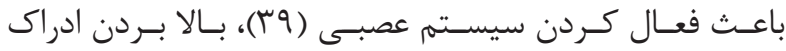

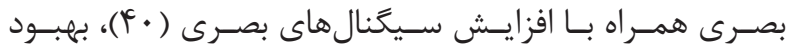

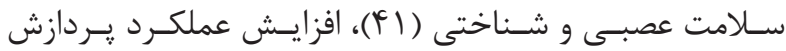

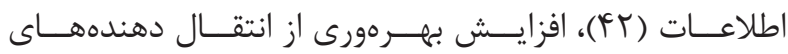

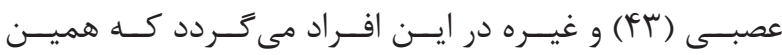

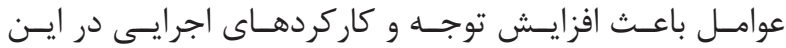
كـود كان مى خــردد.

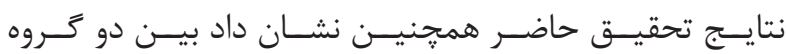

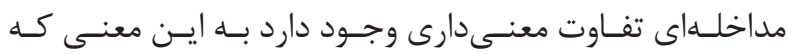

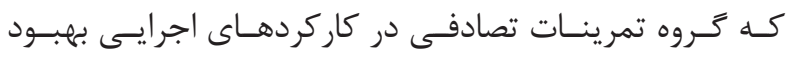

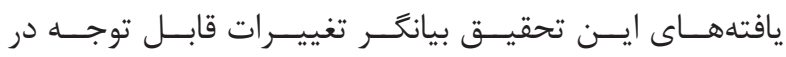

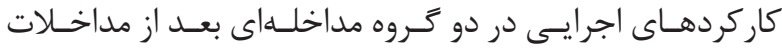

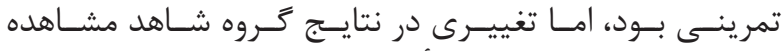

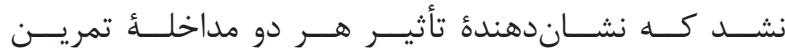

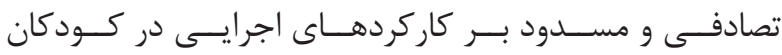

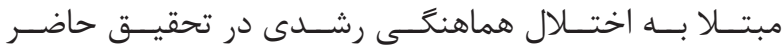

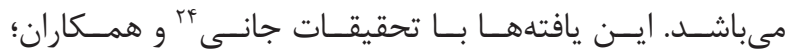

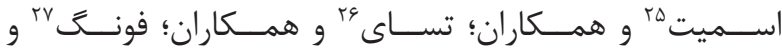

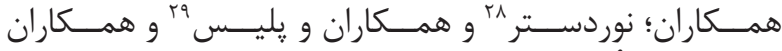

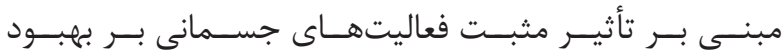

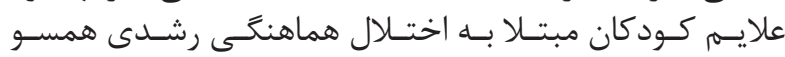
مىباشــد (19، IV، MF-HV)

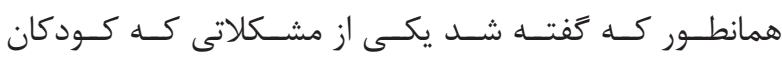

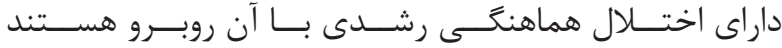

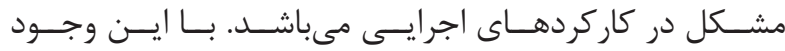

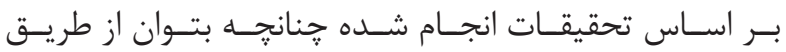

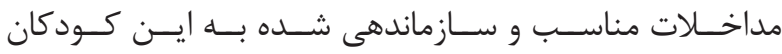

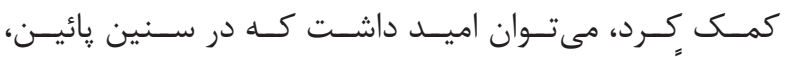

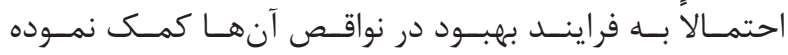

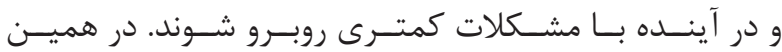

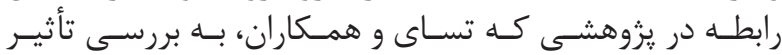

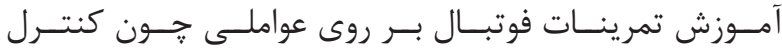

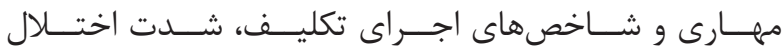

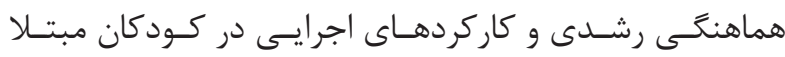

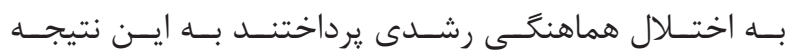

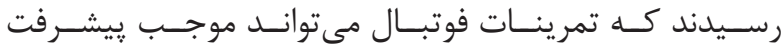

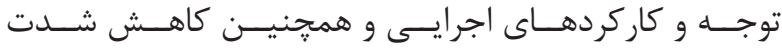

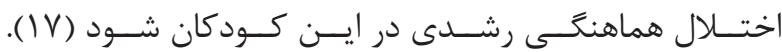

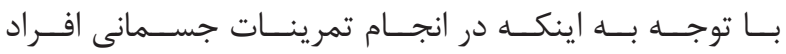

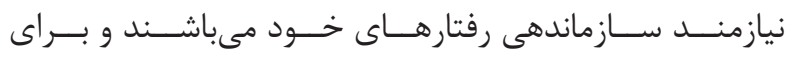

\footnotetext{
${ }^{28}$ Noordstar

${ }^{29}$ Pless

${ }^{30}$ Jones
} 


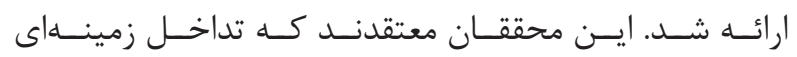

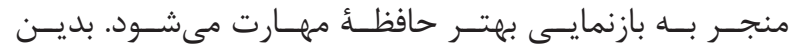

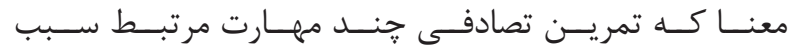

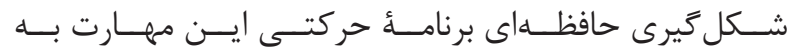

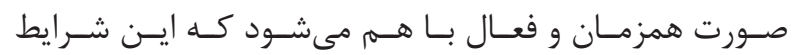

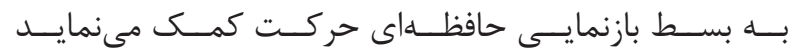

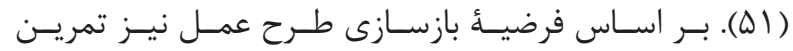

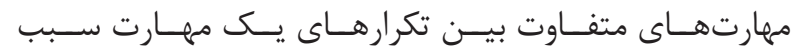

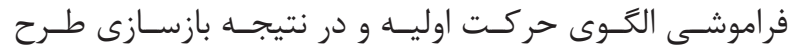

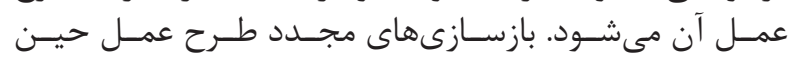

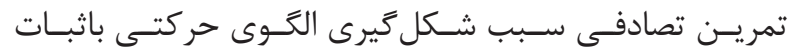

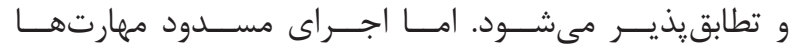

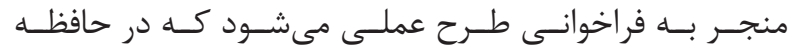

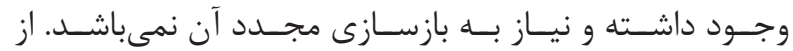

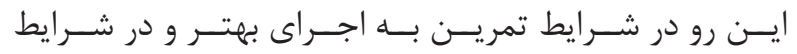

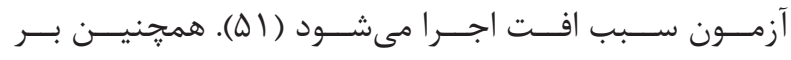

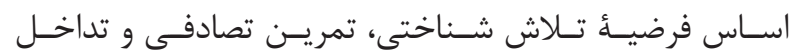

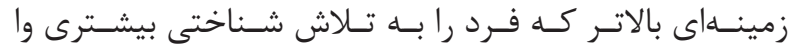

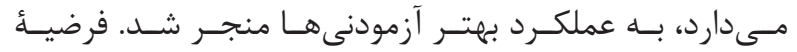

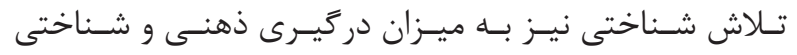

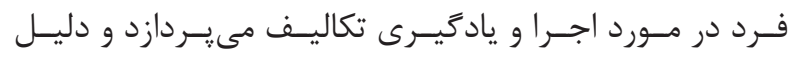

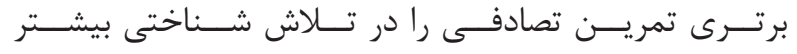

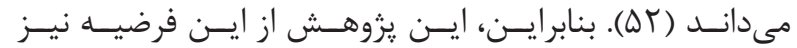

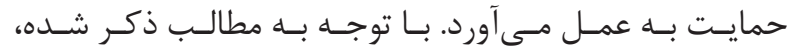

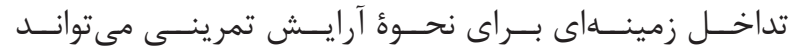

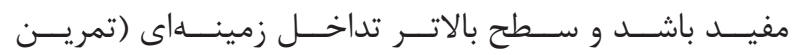

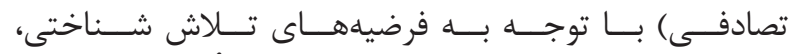

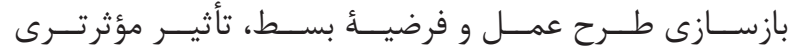

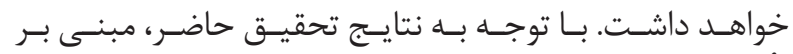

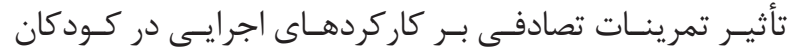

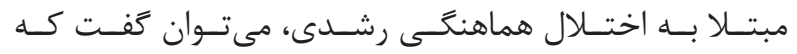

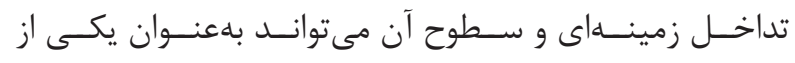

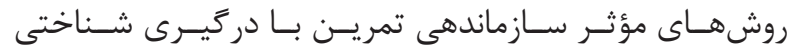

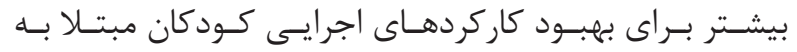

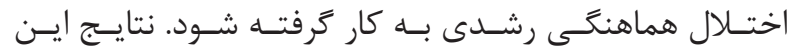

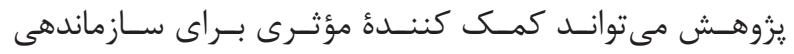

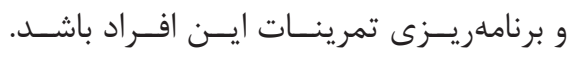

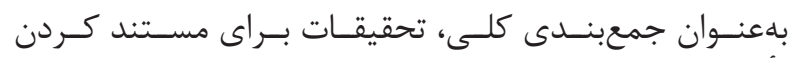

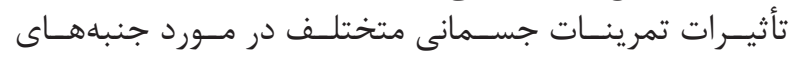

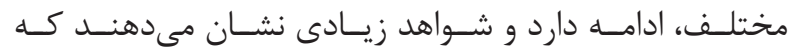

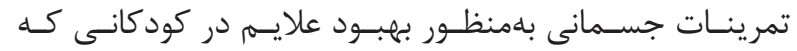

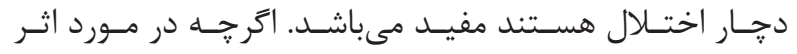

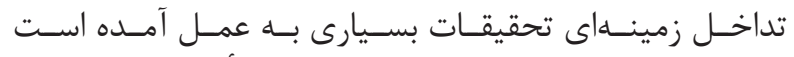

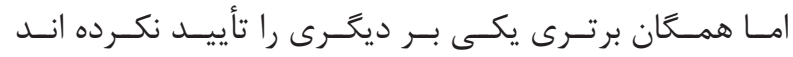

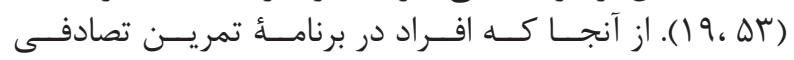

${ }^{31}$ Porter

${ }^{32}$ Russell and Newell

${ }^{33}$ Kurahashi

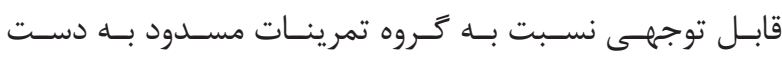

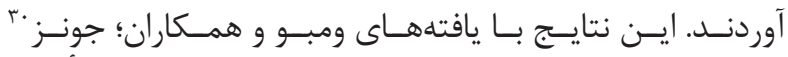

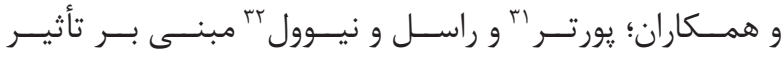

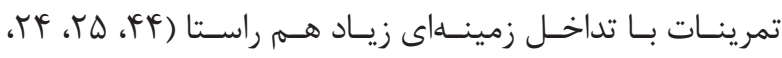

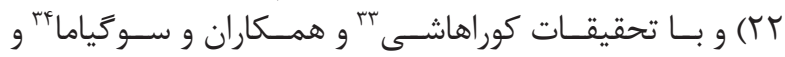

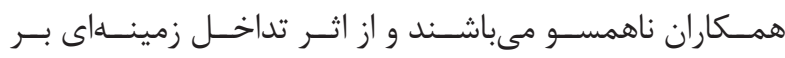

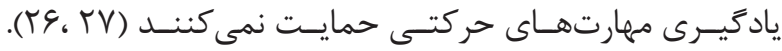

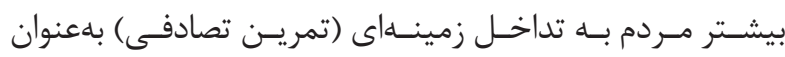

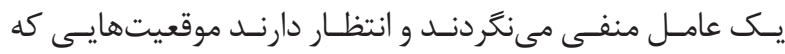

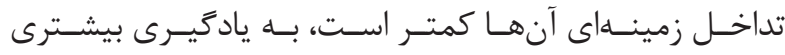

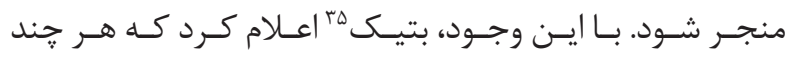

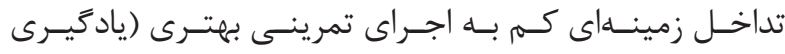

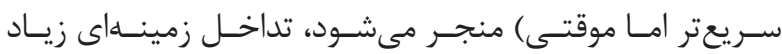

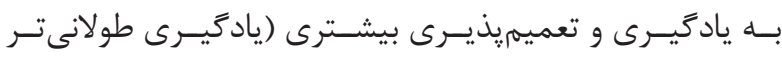

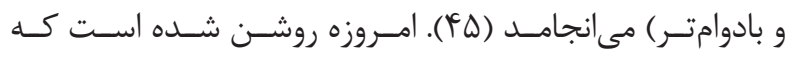

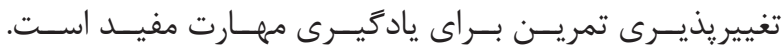

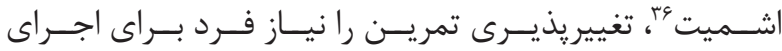

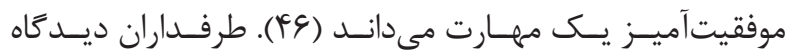

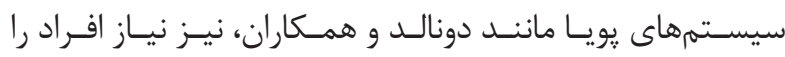

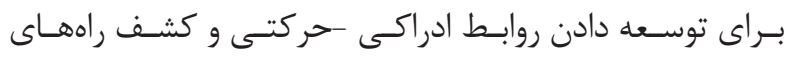

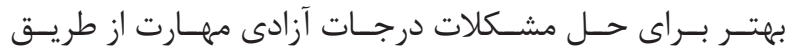

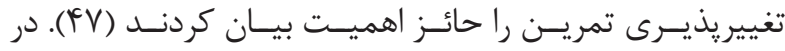

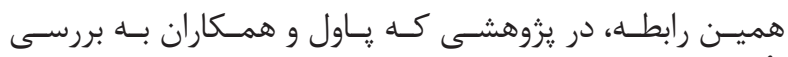

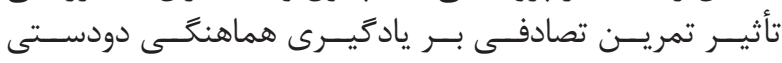

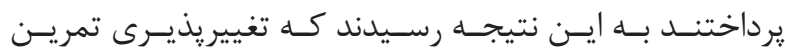

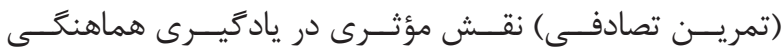

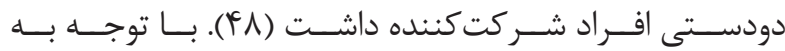

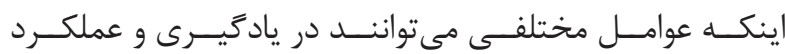

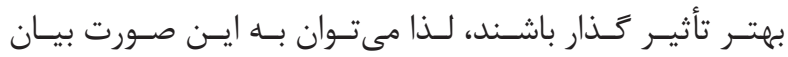

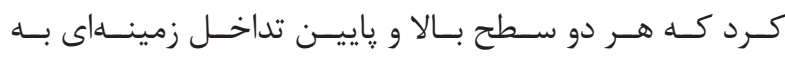

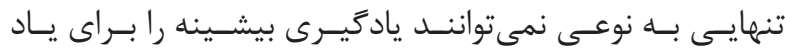

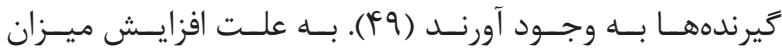

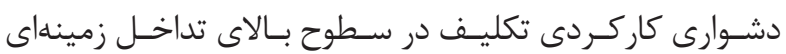

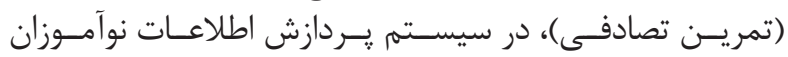

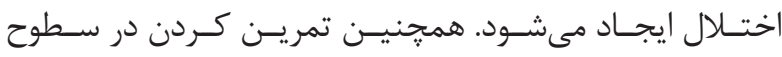

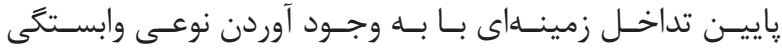

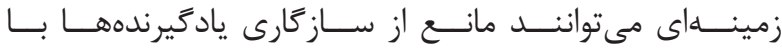

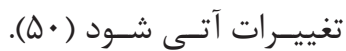

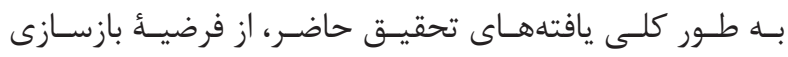

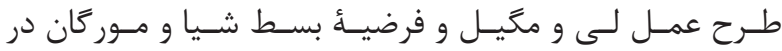

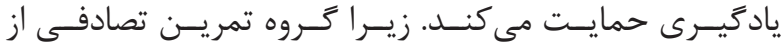

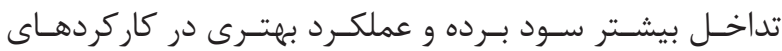

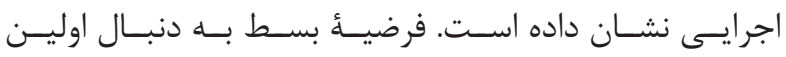

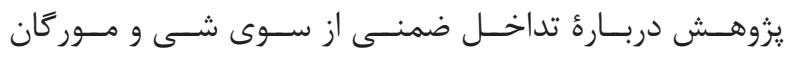

\footnotetext{
${ }^{34}$ Sugiyama

${ }^{35}$ Battig

${ }^{36} \mathrm{Schmidt}$
} 


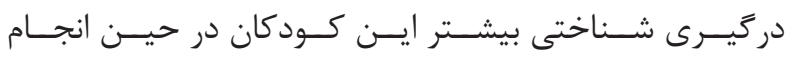

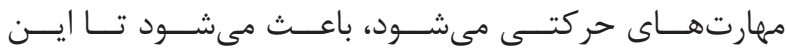

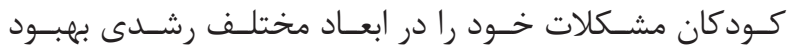

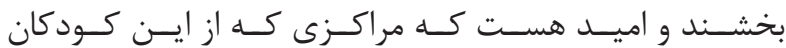

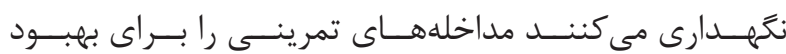

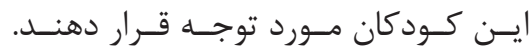

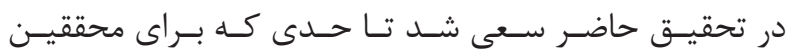

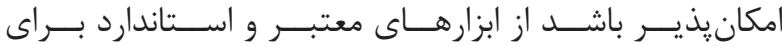

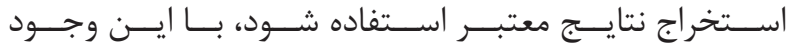

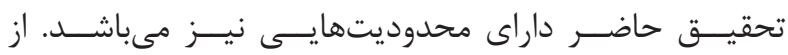

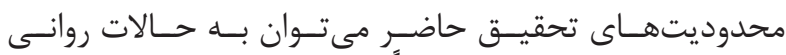

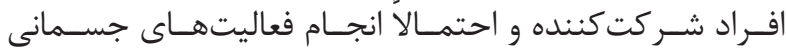

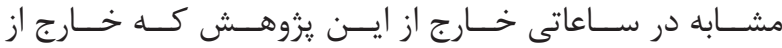

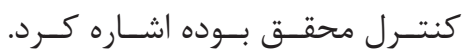
تشكر و قدردانى

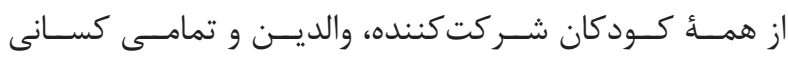

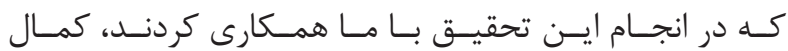

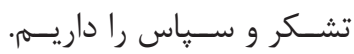

1. Rivilis I, Hay J, Cairney J, Klentrou P, Liu J, Faught BE. Physical activity and fitness in children with developmental coordination disorder: A systematic review. Res Dev Disabil. 2011; 32(3): 894-910.

2. Alloway TP, Archibald L. Working memory and learning in children with developmental coordination disorder and specific language impairment. J Learn Disabil. 2008; 41(3): 251-62.

3. Henderson SE, Henderson L. TowardAnUnderstanding ofDevelopmentalCoordinationDisorder:Terminological and Diagnostic Issues. Neural Plast. 2003; 10 (1-2): 1-13.

4. McLeod KR, Langevin LM, Goodyear BG, Dewey D. Functional connectivity of neural motor networks is disrupted in children with developmental coordination disorder and attention-deficit/hyperactivity disorder. Neuroimage Clin. 2014; 4: 566-75.

5. Kadesjo B, Gillberg C. Developmental coordination disorder in Swedish 7-year-old children. J Am Acad Child Adolesc Psychiatry. 1999; 38(7): 820-8.

6. Baghernia R, Asle Mohammadizadeh M. Prevalence of developmental coordination disorder in iranian 3-to11-year-old children. J Res Rehabil Sci. 2014; 9(6): 1077-99.

7. A American Psychiatric Association. Diagnostic and statistical manual of mental disorders. (DSM-5®): American Psychiatric Pub; 2013. p. 947.

8. Maruff P, Wilson P, Trebilcock M, Currie J.

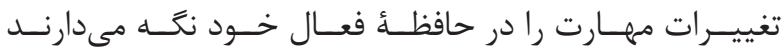

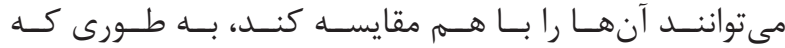

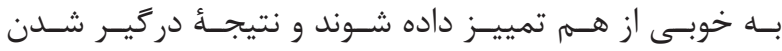

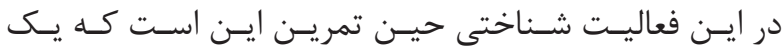

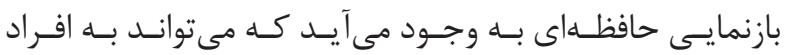

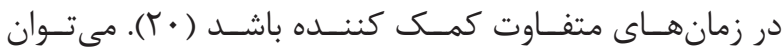

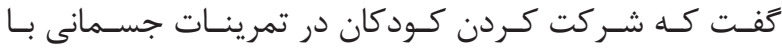

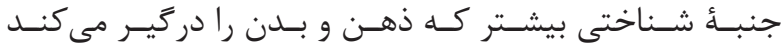

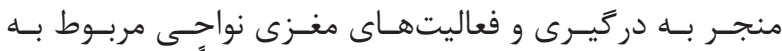

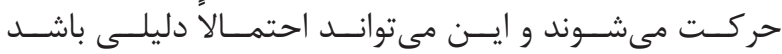

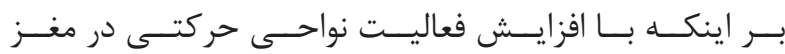

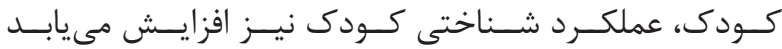

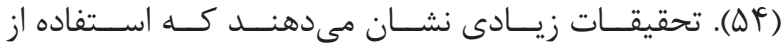

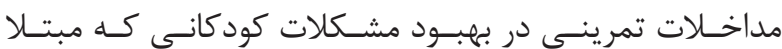

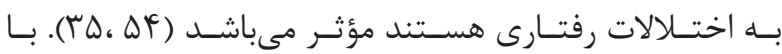

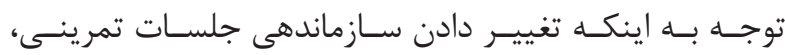

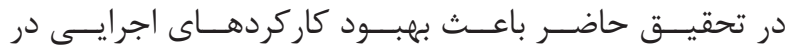

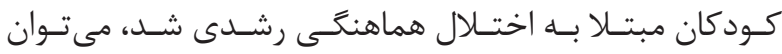

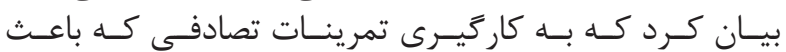

منابع

Abnormalities of imagined motor sequences in children with developmental coordination disorder. Neuropsychologia. 1999; 37(11): 1317-24.

9. Wilson PH, Maruff P, Ives S, Currie J. Abnormalities of motor and praxis imagery in children with DCD. Hum Mov Sci. 2001; 20(1): 135-59.

10. Wilson P, Maruff P, Butson M, Williams J, Lum $\mathrm{J}$, Thomas P. Internal representation of movement in children with developmental coordination disorder: a mental rotation task. Dev Med Child Neurol. 2004; 46(11): 754-9.

11. Schoemaker MM, van der Wees M, Flapper B, Verheij-Jansen N, Scholten-Jaegers S, Geuze RH. Perceptual problems in children with a developmental coordination disorder. Hum Mov Sci. 2001; 20(1-2): 111-33.

12. McClelland MM, Cameron CE, Duncan R, Bowles RP, Acock AC, Miao A, et al. Predictors of early growth in academic achievement: The head-toesknees-shoulders task. Front Psychol. 2014; 5: 599. doi: 10.3389/fpsyg.2014.00599.

13. Becker DR, Miao A, Duncan R, McClelland MM. Behavioral self-regulation and executive function both predict visuomotor skills and early academic achievement. Early Child Res Q. 2014; 29(4): 411-24.

14. Rajendran G, Mitchell P. Cognitive theories of autism. Dev Rev. 2007; 27(2): 224-60. 
15. Barkley RA. Defiant children: A clinician's manual for parent training. New York: Guilford Press. 1987.

16. Jane JY, Burnett AF, Sit CH. Motor skill Interventions in children with developmental coordination disorder: a systematic review and meta-analysis. Arch Phys Med Rehabil. 2018; 9993(18): 30004-2.

17. Tsai C-L, Wang C-H, Tseng Y-T. Effects of exercise intervention on event-related potential and task performance indices of attention networks in children with developmental coordination disorder. Brain Cogn. 2012; 79(1): 12-22.

18. Merbah S, Meulemans T. Learning a motor skill: effects of blocked versus random practice. a review. Psychol Belg. 2011; 51: 15-48.

19. Zetou E, Michalopoulou M, Giazitzi K, Kioumourtzoglou E. Contextual interference effects in learning volleyball skills. Percept Mot Skills. 2007; 104(3): 995-1004.

20. Magill RA. Motor learning and control. $10^{\text {th }}$ ed. McGraw-Hill Education. 2011. p. 496.

21. Schmidt RA, Wrisberg CA. Motor learning and performance. 3rd ed. Human Kinetics. 2004. p. 381.

22. Wambaugh JL, Nessler C, Wright S, Mauszycki SC, DeLong C, Berggren K, et al. Effects of blocked and random practice schedule on outcomes of sound production treatment for acquired apraxia of speech: results of a group investigation. J Speech Lang Hear Res. 2017; 60(6S): 1739-51.

23. Fazeli D, Taheri H, Saberi Kakhki A. Random Versus blocked practice to enhance mental representation in golf putting. Percept Mot Skills. 2017; 124(3): 674-88.

24. Jones K, Croot K. The effect of blocked, random and mixed practice schedules on speech motor learning of tongue twisters in unimpaired speakers. Motor Control. 2016; 20(4): 350-79.

25. Russell DM, Newell KM. How persistent and general is the contextual interference effect? Res Q Exerc Sport. 2007; 78(4): 318-27.

26. Kurahashi A, Leming K, Carnahan H, Dubrowski A. Effects of expertise, practice and contextual interference on adaptations to visuo-motor misalignment. Stud Health Technol Inform. 2008; 132: 225-9.

27. Sugiyama M, Araki M, Choshi K. Order of a 'uniform random'presentation on contextual interference in a serial tracking task. Percept Mot Skills. 2006; 102(3): 839-54.
28. Abedi A, Malekpour MP, Moulavi H, Arizihr Amiri SH. Compare neuropsychological features young children with neuropsychological/ development learning disabilities and normal, preschool. Res Exceptional Children. 2007; 2(10): 38-48.

29. Dehghan M, Karimei N, Tagipourjavan AA, Hasan NattajJelodar F, Zaidabadi F. The effectiveness of rhythmic movement games (weighted) on the rate of executive function in children with neuropsychological learning disabilities. J Learning Disabil. 2012; 2(1): 53-77.

30. Wilson PH. Practitioner review: approaches to assessment and treatment of children with DCD: an evaluative review. J Child Psychol Psychiatry. 2005; 46(8): $806-23$

31. Wilson BN, Crawford SG, Green D, Roberts G, Aylott A, Kaplan BJ. Psychometric properties of the revised developmental coordination disorder questionnaire. Phys Occup Ther Pediatr. 2009; 29(2): 182-202.

32. Afsordeh Bakhshayesh R, Salehi H, Movahedi A, Ghasemi V. The log version of the psychometric properties of coordination impaired motor development in children 6-11 years old. Journal of Psychology Special. 2010; 1(4): 135-61.

33. Moradi H, Sohrabi M, Mones-Tusi. The effect of exercises with contextual interference of training on attention problems in children with developmental coordination disorder. Shahrekord University of Medical Sciences. 2017; 19(5): 46-59.

34. Fong SS, Tsang WW, Ng GY. Taekwondo training improves sensory organization and balance control in children with developmental coordination disorder: a randomized controlled trial. Res Dev Disabil. 2012; 33(1): 85-95.

35. Noordstar JJ, van der Net J, Voerman L, Helders PJ, Jongmans MJ. The effect of an integrated perceived competence and motor intervention in children with developmental coordination disorder. Res Dev Disabil. 2017; 60: 162-75.

36. Pless M, Carlsson M. Effects of motor skill intervention on developmental coordination disorder: a meta-analysis. J Hum Kinet. 2000; 17(4): 381-401.

37. Smits-Engelsman BC, Jelsma LD, Ferguson GD. The effect of exergames on functional strength, anaerobic fitness, balance and agility in children with and without motor coordination difficulties living in low-income communities. Hum Mov Sci. 2017; 55: 327-37.

38. Ball MF. Developmental coordination disorder: hints and tips for the activities of daily living. Jessica Kingsley 


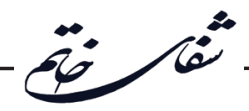

Publishers. 2002.

39. Eimer M, Van Velzen J, Gherri E, Press C. Manual response preparation and saccade programming are linked to attention shifts: ERP evidence for covert attentional orienting and spatially specific modulations of visual processing. Brain Res. 2006; 1105(1): 7-19.

40. Norton DJ, McBain RK, Öngür D, Chen Y. Perceptual training strongly improves visual motion perception in schizophrenia. Brain Cogn. 2011; 77(2): 248-56.

41. Velikonja O, Čurić K, Ožura A, Jazbec SŠ. Influence of sports climbing and yoga on spasticity, cognitive function, mood and fatigue in patients with multiple sclerosis. Clin Neurol Neurosurg. 2010; 112(7): 597-601.

42. Audiffren M, Tomporowski PD, Zagrodnik J. Acute aerobic exercise and information processing: energizing motor processes during a choice reaction time task. A Acta Psychol (Amst). 2008; 129(3): 410-9.

43. Leung L-Y, Tong K-Y, Zhang S-M, Zeng X-H, Zhang K-P, Zheng X-X. Neurochemical effects of exercise and neuromuscular electrical stimulation on brain after stroke: a microdialysis study using rat model. Neurosci Lett. 2006; 397(1): 135-9.

44. Porter JM. Systematically increasing contextual interference is beneficial for learning novel motor skills. Louisiana State University LSU Digital Commons. 2008.

45. Batting WF. "Facilitation and interference". EA. Bilodeau. Acquisition of skill. New York: Academic
Press. 1966. p. 215-44.

46. Schmidt RA. A schema theory of discrete motor skill learning. Psychological Review. 1975; 82(4): 225.

47. Brady F. A theoretical and empirical review of the contextual interference effect and the learning of motor skills. Quest. 1998; 50(3): 266-93.

48. Pauwels L, Swinnen SP, Beets IA. Contextual interference in complex bimanual skill learning leads to better skill persistence. PloS One. 2014; 9(6): e100906.

49. Magill RA, Hall KG. A review of the contextual interference effect in motor skill acquisition. Hum Mov Sci. 1990; 9(3): 241-89.

50. Magill RA, Anderson DI. Motor learning and control: concepts and applications. New York: McGrawHill. 2007. p. 482.

51. Latash ML, Lestienne F. Motor control and learning: Springer; 2006.

52. Lee TD, Swinnen SP, Serrien DJ. Cognitive effort and motor learning. Quest. 1994; 46(3): 328-44.

53. Fialho J, Benda R, Ugrinowitsch H. The contextual interference effect in a serve skill acquisition with experienced volleyball players. Journal of Human Movement Studies. 2006; 50(1): 65-77.

54. Zwicker JG, Missiuna C, Harris SR, Boyd LA. Developmental coordination disorder: a review and update. Eur J Paediatr Neurol. 2012; 16(6): 573-81. 